\title{
Transcriptional response to kairomone exposure in Daphnia magna. A candidate gene approach
}

Murat Telli ( $\nabla$ mtellim@gmail.com )

Abant Izzet Baysal Universitesi Fen Edebiyat Fakultesi https://orcid.org/0000-0001-5546-0379

Donna M. Gordon

Mississippi State University

Ercan Selçuk Ünlü

Abant Izzet Baysal Universitesi Fen Edebiyat Fakultesi

Research article

Keywords: Daphnia magna, microRNAs, mRNA transcripts, Fish kairomone

Posted Date: January 3rd, 2020

DOI: https://doi.org/10.21203/rs.2.20016/v1

License: (1) This work is licensed under a Creative Commons Attribution 4.0 International License.

Read Full License 


\section{Abstract}

Background: Daphnia (Brachiopoda, Cladocera) is a well-studied model organism providing unparalleled opportunity to test epigenetic regulation of predator avoidance mechanisms in aquatic ecosystems. The discovery of regulatory functions for microRNA molecules and recently described miRNA profiles of Daphnia make it an ideal system to probe for posttranslational regulatory mechanisms mediated by kairomone released by predatory fish. However, despite a number of studies that focused on mRNA transcript level differences, no miRNA studies associated with kairomone exposure have been reported.

Results: Exposing D. magna to fish kairomone from birth to the first reproduction was found to result in the differential expression of the four miRNAs tested: miR-7, miR-34, miR-317, and miR-375. Normalized transcript levels for each miRNA were found to vary across the exposure period with no clear conserved pattern of expression despite functional target analyses by GO, COG and KEGG indicating that predicted miRNA target genes are likely involved in related biological activities. Analysis of six mRNA transcripts (Hsp70, Hsp90, actin, AKT, GYS and IGFR), identified in previous studies as kairomone-mediated genes in Daphnia magna, were also carried out. Similar to that obtained for miRNAs, the mRNA transcript levels showed varying degrees of temporal regulation across the exposure time course with the two heat shock transcripts exhibiting elevated levels at early and late time points of kairomone exposure while the AKT, GYS, and IGFR transcripts had an general decrease in expression during the first 96 hours.

Conclusions: Differential mRNA expression data supports the premise of an ecological trade-off between the cost of general biological processes and that of survival under long-term kairomone stress. Transcript levels for the four miRNAs tested were found to vary across developmental time with kairomone exposure which suggests that they may have a role in regulating morphological, behavioral or physiological responses by altering target gene expression. These studies lay the foundation for future work aimed at linking miRNAs and their target transcripts to changes in the signaling events that govern Daphnia response to kairomone specific stress.

\section{Background}

The small planktonic crustacean, Daphnia, is a model organism often at the center of studies aimed at characterizing the ecology of freshwater food webs. Because of this, there are a large number of datasets available that provide both genomic and ecological information. Daphnia also have unique biological attributes that contribute to their broad utility including exhibiting morphological, behavioral and physiological alterations in response to chemical or physical cues released by different sources of biotic and abiotic stressors including food availability, temperature, and predator presence (Reynolds 2011). Detection of cues released by common predators such as fish (i.e. kairomones) leads to a quick behavioral alteration that takes place within a few hours of exposure. In Daphnia, this response is called the diurnal vertical migration (DVM). As part of the DVM response, Daphnia move into the lower layer of a lake during the day to avoid predation (Meester et al. 1999). Such predator avoidance strategy requires the engagement of quick and effective multi-defense mechanisms against predation pressure, food 
limitation, low temperature, and darkness. Given the short timeframe required for these changes, epigenetic modifications have been predicted to play a role as a regulatory mechanism (Harris et al. 2012). With their well characterized life cycle, ease of laboratory-based cultivation, clonal reproductive capabilities, and the availability of whole genome sequence data and miRNA profiles (Colbourne et al. 2011, Ünlü et al. 2015, Hearn et al. 2018, Coucheron et al. 2019), Daphnia are ideal candidate organisms to probe for epigenetic regulatory mechanisms responsible for directing biological responses to changes in environmental cues.

As non-coding transcripts, microRNAs have been implicated as regulators of gene expression changes induced by a wide range of cellular conditions including aging, exposure to environmental agents, various diseased states from diabetes to cancer, and host-parasite interactions (Hu et al. 2018; Price 2014; Judice et al. 2016). Typically, these short ribonucleotide molecules work at the post-transcriptional level to target cellular mRNA transcripts for translational inhibition or degradation through their assembly with the RNAinduced silencing complex, or RISC (Carthew and Sontheimer 2009). Specifically, the miRNA-RISC complex targets mRNA transcripts that exhibit some level of sequence complementary with the 22 nucleotide long miRNA. In Daphnia, interest in identifying the biological roles for miRNAs have increased due to their connection to multiple stress-related cellular mechanisms. For example, Daphnia specific stress responsive miRNAs associated with exposure to heavy metal (Chen et al. 2016), changing nutritional conditions, (Hearn et al. 2018) and ageing (Hu et al. 2018) have recently been identified. To date, no miRNA studies linking abiotic stressors such as kairomone to changes in gene regulation have been reported. However, changes in mRNA transcript levels for Daphnia in the presence of kairomone have been studied using transcriptomics, microarray, and target gene approaches (Pijanowska and Kloc 2004; Pauwels et al. 2005; Jansen et al. 2013; Schwarzenberger et al. 2009; Miyakawa et al. 2010).

Results from these studies indicate a shift in energy use from reproduction to survival for adults in the presence of kairomone. Specifically, genes involved in energy metabolism were found to be downregulated in adult Daphnia (Jansen et al. 2013), but not in juvenile stage individuals (Miyakawa et al. 2010). This is consistent with the finding that only adult Daphnia show DVM behavior in the presence of kairomone. Candidate gene approaches have identified several genes involved in kairomone-induced gene expression including heat shock protein (HSP) genes, which are widely known for their role of defense against different types of stress, and actin and tubulin genes, which are involved in cytoskeletonbased reorganization processes (Pijanowska and Kloc 2004; Pauwels et al. 2005). Focusing on candidate genes involved in endocrine, morphogenic, or neural regulation, Miyakawa et al. (2010) identified 21 transcripts that were upregulated in a kairomone-dependent manner in the first instar stage of juvenile D. pulex and not during the prior embryonic stage of development. As defense characteristics are apparent in later stages of instar development, upregulation during the first-instar stage is proposed to establish the induction of this defense morphology.

Although there are a number of reports characterizing mRNA transcript level changes in response to kairomone, to our knowledge there are no studies that have focused on miRNA profiles for Daphnia under these conditions. The goals of this study were therefore multifold. First, to use a quantitative PCR 
approach to measure the relative expression level of select mRNA transcripts previously shown to be influenced in a kairomone-mediated manner. Second, to validate the expression of miRNAs previously identified by small RNA sequencing studies (Ünlü et al. 2015) and to characterize changes in these miRNA transcript levels in response to kairomone exposure. Finally, to take a biocomputational approach towards identifying potential mRNA targets for these miRNAs with the goal of predicting the functional consequence alterations in these small regulatory RNAs may have on defense response across Daphnia development. Overall, data from this small-scale analysis of D. magna miRNA transcripts will provide a platform for additional efforts aimed at identifying a role for miRNAs in predator avoidance strategies.

\section{Results}

\section{Life-history}

As a strategy to avoid fish predation, field acquired Daphnia clones exposed to fish kairomone reproduce at an earlier age and with a higher offspring number when compared to an unchallenged cohort (Stibor 1992; Tollrian 1995; Dodson and Havel 1998). However, reports differ as to whether a similar response occurs with Daphnia maintained long term in a laboratory setting, such as those obtained from a commercial source (Pijanowska 2004). In our hands, kairomone-exposed D. magna obtained from a commercial vendor started producing eggs $24 \mathrm{~h}$ earlier than control groups with the age of first reproduction for control and fish kairomone-exposed individuals at $144 \mathrm{~h}$ and $120 \mathrm{~h}$, respectively. At the end of the experiment, the mean number of juveniles produced per female for control and kairomoneexposed groups were $5.07 \pm 1.42$ and $9.82 \pm 2.71$, respectively. Therefore, similar to data obtained using field obtained Daphnia, the kairomone-exposure Daphnia used in our experiments resulted in the production of juveniles earlier and in significantly more numbers than control groups $(P<0.02, t$ test).

\section{Relative mRNA expression}

Prior work varied in the predator derived-kairomone, clones of D. magna used, and exposure methodology. To place our work in the context of current findings, we analyzed changes in the level of six mRNA transcripts often targeted as reporters for stress-based kairomone exposure: Hsp70, Hsp90, and actin (Pijanowska, 2004, Otte 2014), and the energy metabolism genes AKT, GYS and IGFR (Jansen et al. 2013 and Miyakawa et al. 2010) (Figure 1). A qPCR-based approach was carried out on RNA isolated from individuals exposed to vertebrate kairomone for $24,48,96$, and 144 hours. For data normalization, glyceraldehyde 3-phosphate dehydrogenase (GAPDH) transcript levels were used as several reports have indicated stability under similar experimental conditions (Schwarzenberger 2009, Miyakawa 2010).

Transcript levels for Hsp70 and Hsp90 relative to unexposed Daphnia at $\mathrm{t}_{(0)}$, were found to increase 3fold following 24 hours of exposure but returned to basal levels at the 48-h and 96-h time points (Figure 1). Interestingly, a second upregulation in relative transcript levels were recorded for both HSPs at 144 hours, a time point that followed the average age of the first reproduction for these organisms (120 
hours). This is in contrast to actin transcript levels which showed a minor decrease in the presence of fish kairomone at 24, 48 and 96 hours but a more substantial 7-8 fold decline with 144 hours of exposure. Our data also indicated a decrease in the mRNA transcript level for three insulin signaling pathway genes (ATK, GYS, and IGFR) between 24 and 96 hours of kairomone exposure with a return to basal level at 144 hours exposure.

\section{Relative miRNA expression}

The identification of miRNAs in D. magna is relatively recent (Ünlü et al., 2015) and only two reports have aimed to identify changes in miRNA expression levels, one focusing on calorie restriction and ageing (Hearn et al., 2018) and a second looking across life stages (Coucheron et al., 2019). To determine a potential role for miRNAs in regulating gene expression in response to kairomone exposure, we took a targeted approach, monitoring the transcript levels of four miRNAs: miR-7, miR-34, miR-317, and miR-375. These miRNAs have each been proposed to function broadly during development by targeting cell cycle progression, as well as regulating stress response, immune response, morphological development and general metabolic pathways, respectively (Li et al., 2009, Isik et al., 2016, Jain et al. 2015).

By qPCR, all miRNAs were found to be differentially expressed in response to kairomone exposure (Figure 2). miR-375 and miR-317 shared a similar overall expression profile with significantly higher miRNA levels at 24-h and 144-h of exposures. In contrast, miR-7 levels were significantly downregulated at the later time points. miR-34 transcript levels exhibited the most variation with a 2-fold increase at 24-h followed by a general trend that suggested a decrease over all later time points.

\section{mRNA - miRNA target prediction}

To assess the potential functional roles for these miRNAs, a bioinformatics approach was utilized to identify mRNA targets. As an annotated genome data for Daphnia magna is limited, putative mRNA targets were identified using 3' UTR data available for Drosophila. To enhance confidence in the prediction, targets with 8- and 7-nucleotide complementarity were selected. Using this approach, a total of 183 putative targets were identified for miR-7, 212 for miR-34, 201 for miR-317, and 287 for miR-375. A detailed list of putative miRNA targets, their site type, and context scores are presented in Additional Table 1. As mRNA targets for three of the four miRNAs were previously predicted in Drosophila (Stark et al. 2003), we compared these targets with those identified using $D$. magna miRNAs. For miR-7, miR-34, and miR-317 we found overlapping matches for 47, 34, and 48 target genes, respectively (Stark et al., 2003). 
Potential target genes were classified into different functional classes based on their GO, COG, and KEGG annotation data. Outside of proteins with unknown function, COG analyses suggest that many of the predicted target genes function in cellular signaling activities, transcription, and diverse translational regulatory mechanisms including chaperone activity (Additional Figure 1). GO data supports the COG analysis with miR-7, miR-317, and miR-375 targets mapping heavily to the nucleus, while miR-34 targets are found in the plasma membrane in addition to having a nuclear enrichment (Additional Figure 2). KEGG pathway distribution of predicted genes indicate a role in "Environmental information processing" including signal transduction and signaling activities for selected miRNAs (Additional Figure 3 ). These patterns of distribution suggest that these miRNAs have overlapping and specific roles in targeting genes related to molecular signaling. However, the pathways targeted do differ with mir-34-5p predicted to map to membrane related pathways while the other three miRNAs are predicted to control intracellular signaling pathway components.

\section{Discussion}

This work reports the impact kairomone exposure has on gene expression for select mRNA and miRNA transcripts in Daphnia magna as determined by qPCR analysis. Target transcripts were selected based on their predicted role in influencing various activities associated with predatory avoidance mechanisms including regulating cellular stress response and energy metabolism. Results demonstrate changes to key mRNA transcript expression levels with kairomone exposure that can be associated with reallocation of metabolic resources over time. Changes to miRNA transcript levels, along with computation-based predictions aimed at identifying potential mRNA targets, also support this interpretation.

As ATP-dependent chaperones involved in protein folding, heat shock proteins (HSPs) are required for the maintenance of cellular homoeostasis following exposure to internal and/or external stress inducing conditions (Sorensen et al. 2003; Kragel 2002; Haap and Köhler 2009). A number of prior reports have linked HSP activities to the response of kairomone-exposed Daphnia. For example, Otte et al. (2014) and Pijanowska and Kloc (2004) reported a significant decrease in various HSP proteins (Hsp40, Hsp60, Hsp70, Hsp75) in Daphnia exposed to kairomone from the period of birth to the first reproduction. However, a study by Pauwels et al. (2005) found that levels of Hsp60 protein increased over the first 6 hours of exposure before returning to basal levels after 24 hours, although there were some clonal differences in the degree of induction. In contrast, Effertz and Elert (2014) reported no change in Hsp10, Hsp70, or Hsp90 transcript levels within the first 4 hours of exposure to kairomone. Our findings demonstrate a parallel fluctuation in Hsp70 and Hsp90 transcript levels over the 144 hour kairomone exposure time, with significantly higher levels of each transcript at the earliest (24 h) and latest (144 h) time points of testing.

This varying expression pattern for the HSPs may be a result of an ecological trade-off between the cost of chaperone protein expression and the biological requirements of Daphnia for reproductive success under stress conditions. As HSP protein expression is generally regarded as an energy demanding process, continued expression under stress conditions may be unsustainable (Krebs and Feder 1997; 
Silderman and Tatar 2000). Therefore, the early elevation in HSPs may work to counteract the biological consequences of exposure to the initial stressor. However, with continued exposure to stress, a reduction in HSP expression may be favored to save resources for critical developmental and reproductive processes in Daphnia. The return to elevated levels following reproduction may then represent another reshuffling of resource allocation to ensure survival.

Several reports have also linked actin to the defense mechanism of Daphnia species, although the impact of kairomone on actin abundance is contradictory. Pijanowska and Kloc (2004) reported a decrease in actin protein levels in D. magna exposed to vertebrate (fish) or invertebrate (Chaoborus) predator kairomone. This is in contrast to proteomic analyses carried out by Otte et al. (2014) that found an increase in actin for $D$. magna exposed to the invertebrate Triops kairomone. At the transcript level, actin mRNA expression levels have been reported to moderately differ based on the predator kairomone tested: elevated with fish kairomone but decreased in individuals exposed to Chaoborus kairomone (Schwarzenberger et al. 2009). In contrast to the data acquired by Schwarzenberger et al. (2009), we found a continued decrease in actin transcript levels over the 144 hour exposure time course. It is unclear what may contribute to these opposing findings, although differences in qPCR data analysis were identified, most notably their use of three reference genes (GAPDH, SDH, and TBP) for target gene normalization versus our analysis using only one (GAPDH). It is also possible that variability in kairomone exposure times may be a contributing factor especially given our findings of only a minor decrease in actin transcript levels up to 96 hours of kairomone exposure. In the future, analysis of actin transcript levels for samples collected between 96 - and 144 hours of exposure, which are likely to align with Daphnia maturity, may help to clarify this issue.

Insulin-signaling pathways play an important regulatory role in controlling critical energy functions such as glucose and lipid metabolism as well as developmental processes including body size, morphogenesis, and allometry in many animal species (Badisco et al. 2013). To this end, we analyzed the expression of three genes related to insulin-signaling: the insulin-like growth factor receptor, IGFR, the intracellular signaling serine/threonine kinase, AKT, and the glycogen synthase enzyme, GYS. All three transcripts exhibited an overall decrease in expression during the first 48- to 96 hours. Since these experiments were carried out under nutrient replete conditions, it is reasonable to conclude that the reduction in ATK, IGFR, and GYS mRNA transcript levels are likely a result of kairomone exposure. Therefore, these findings suggest a shift in energy usage away from development and towards biological activities required to initiate and maintain predator avoidance strategy such as DVM behavior. As a DVM response requires survival under the unfavorable conditions such as those found in the hypolimnetic layer of a lake, this response is likely to impart a high-energy demand to maintain homeostasis. It should be noted however, that Miyakawa et al. (2010) and Rozenberg et al. (2015) both reported an upregulation of insulin-signaling pathway genes in an early juvenile stage of D. pulex development in the presence of kairomone. These seemingly contradictory findings may be explained by an absence of a DVM response for juvenile daphnids as their small size likely protects them from visual predatory fish. Therefore, the differences in expression profile for insulin signaling genes between juvenile and adult stages may indicate a shift in energy usage away from growth and towards DVM activities. 
With the identification of miRNAs as post-transcriptional regulators of cellular mRNA levels, they have the potential to quickly and efficiently mediate changes to biological pathways often making them the focus of studies investigating developmental events in Daphnia. For example, Hearn et al. (2018) took an RNA sequencing approach to characterize changes in miRNA levels across generational time. Although they report no differences in miRNA levels, they did identify 39 previously unidentified miRNAs. Looking at distinct developmental stages (juvenile, subadult, and adult), Coucheron et al. (2019) identified 66 miRNAs that were present across all developmental time points, with individual transcripts varying in their patterns of abundance. In this report, the relative levels of four miRNAs were analyzed by qPCR in Daphnia exposed to kairomone for a period from birth to the first reproduction time of maturity. All four miRNA transcripts were found to be impacted by kairomone exposure, and with broad grouping, could be placed into two categories: those that had elevated levels (miR-375 and miR-317) and those that had lower than basal levels of transcript (miR-7 and miR-34). Although changes in transcript levels for these miRNAs may be viewed as moderate, statistically significant changes were identified. It is also important to note that a single miRNA transcript loaded into RISC can influence the stability/translation of multiple mRNA targets which would result in an amplification of any moderate changes in miRNA levels. An additional consideration is the fact that fold changes were determined by comparison to unexposed individuals at $t_{0}$. The relative miRNA levels are therefore likely a reflection of the combined impact of kairomone exposure and developmental programming. This conclusion is supported by the recent RNA sequencing results from Coucheron et al. (2019) showing that miRNA transcript abundance varies across developmental stages in D. magna. Specifically, they report that transcript levels for miR-375 increased as development progressed (juvenile to subadult to adult), while transcript levels for miR-317, miR-34, and miR-7 were least abundant in adult Daphnia and highest in the prior subadult stage (Coucheron et al., 2019). Interpreting our findings within this context suggests that kairomone exposure has a suppressive effect on the miRNAs analyzed, with its largest suppressive effect on 48-h and 96-h exposed individuals.

\section{Conclusions}

Among the six mRNAs transcript analyzed in the present study, Hsp70, Hsp90 and actin have been commonly targeted to assess kairomone mediated stress regulation in Daphnia. Our results support the conclusion that HSPs may be temporally regulated in the context of an ecological trade-off between the associated cost of general biological processes and the benefit of survival under long-term stress conditions. Differential expression results for three energy metabolism-related genes, ATK, IGFR, and GYS, may indicate additional ecological trade-offs with regards to energy requirements and survival in long term kairomone stress. Interestingly, the downregulation of ATK, IGFR and GYS genes at 24 hours exposure was followed by a decrease in HSP expression at 48 hours. As HSP activity would impose a high-energy demand required for the maintenance of cellular homoeostasis, it is tempting to speculate that these chaperones may be co-regulated with that of select energy metabolism genes at a posttranscriptional level. 
The qPCR approach to monitor the transcript level of four miRNAs, miR-7, miR-34, miR-317, and miR-375, identified a potential role for these regulatory RNAs in kairomone mediated predator avoidance in Daphnia. Although not directly tested in this study, when changes in miRNA abundance across developmental stages are considered (Coucheron et al., 2019), the accumulated data appears to suggest that kairomone exposure may suppress miRNA transcript expression. It is tempting to speculate that this may contribute to an altered mRNA target transcript abundance that could result in the earlier development of Daphnia that is seen with predator kairomone exposure. In addition to characterizing changes in miRNAs transcript levels, this study also confirmed endogenous expression of these miRNAs, including miR-375, a microRNA that was identified in our prior work (Ünlü et al. 2015) but was absent from the study of Hearn et al. (2018). Recent work has also indicated the presence of miR-375 transcripts in Daphnia (Coucheron et al., 2019). A significant number of the predicted mRNA targets we found to overlap between miRNAs from Drosophila and D. magna (Stark et al., 2003). The functional classes for the predicted mRNA targets as determined by GO, COG and KEGG analyses suggest that target genes are primarily involved in signal transduction or cellular communication related activities with miRNAs having overlapping yet specific contributions towards response regulation. In general, cellular signaling plays a critical role in homeostasis to reorganize biochemical responses with the goal of adapting cellular activities to new environmental conditions (Hotamisligil and Davis 2016). Specifically, both heat shock proteins (Nollen and Morimoto 2002) and proteins involved in insulin signaling (Hardie 2012) are influenced by multiple signaling pathways to control proliferation and development under different environmental stress conditions. Future work focusing on potential regulatory roles for miRNAs in modulating the signaling cascades identified in this work may be an informative way to uncover molecular responses of Daphnia to kairomone specific environmental stress mechanisms.

\section{Methods}

\section{Experimental organisms}

A single clone of Daphnia magna provided by Carolina culture collection (USA), was reared in the laboratory at $23^{\circ} \mathrm{C}$ and in $18 / 6 \mathrm{~h}$ light dark photocycle conditions in $2 \mathrm{~L}$ of Aachener Daphnien Medium (ADaM) (Klüttgen et al. 1984) containing $\mathrm{CaCl}_{2}{ }^{\star} 2 \mathrm{H}_{2} \mathrm{O}, 0.27 \mathrm{~g} / \mathrm{L} ; \mathrm{NaHCO}_{3}, 0.056 \mathrm{~g} / \mathrm{L} ; \mathrm{SeO}_{2}, 0.007 \mathrm{mg} / \mathrm{L}$; sea salt, $0.33 \mathrm{~g} / \mathrm{L}$ in deionized water at a density of 20 individuals per liter. The Daphnia used in these experiments were derived from a clone maintained in a laboratory setting for at least two years. Cultures

were fed daily by $2 \mathrm{mg} \mathrm{C}_{\text {part }} / \mathrm{L}$ of Scenedesmus obliquus (SAG-276-3a) green algae. ADaM medium was enriched by bubble aeration for several days before use and culture medium was renewed twice a week. The third clutch juveniles between 0 to $24 \mathrm{~h}$ old were used for all experiments.

\section{Experimental design}

To obtain fish kairomone conditioned water, 3 cichlid fish $6 \mathrm{~cm}$ in length were starved for 24 hours before the experiment, then held for 24 hours in $4 \mathrm{~L}$ of aerated and filtered $A D a M$ medium. The resulting fish water was diluted 4 times with new ADaM medium to achieve a kairomone concentration of 3 fish in $16 \mathrm{~L}$ 
and filtered $(0.45 \mu \mathrm{m})$ before use as kairomone-enriched medium (De Meester and Cousyn 1997). Control treatments contained only aerated and filtered $(0.45 \mu \mathrm{m})$ ADaM medium. Sixty individuals of new born D. magna neonates were exposed in a $2 \mathrm{~L}$ glass container to kairomone-enriched ADaM medium or only ADaM medium from birth until maturity. All treatments were run in triplicate. Neonates were fed with $2 \mathrm{mg}$ $\mathrm{C}_{\text {part }} / \mathrm{L}$ per liter of $\mathrm{S}$. obliquus and the medium changed daily. The age of the first maturation was determined at the day when the first clutch was visible for all individuals. The reproduction rate of experimental groups was determined by the number of the first clutch of neonates at the end of the experiment.

\section{Total RNA extraction and reverse transcription}

Randomly selected individuals were collected for gene expression analyses at the beginning $\left(\mathrm{t}_{0}-75\right.$ individual) and after 24 ( $\mathrm{t}_{24}-19$ individuals), 48 ( $\mathrm{t}_{48}-19$ individuals), 96 ( $\mathrm{t}_{96}-10$ individuals), and 144 $\left(t_{144}-7\right.$ individuals) hours of exposure. For the $t_{0}$ sampling, all of the neonates were pooled in a $5 \mathrm{~L}$ glass container and 75 individuals were randomly collected with a wide mouth pipet, immediately plunged in liquid nitrogen for the euthanasia, and stored at $-80^{\circ} \mathrm{C}$ until RNA extraction. $\mathrm{t}_{0}$ samples were used for the initial time sampling for both kairomone and control groups. Total RNA was extracted using the Trizol ${ }^{8}$ extraction method (Invitrogen, USA) according to the manufacturer's protocol. After sample homogenization in $1 \mathrm{~mL}$ of Trizol, total RNA was precipitated with the addition of $0.5 \mathrm{ml}$ of $100 \%$ isopropyl alcohol with subsequent RNA phase separation from chloroform at $12000 x \mathrm{x}$ centrifugation for 20 min. After a DNase treatment (TURBO ${ }^{\text {TM }}$ Ambion Life Technologies, USA), RNA quality and integrity were checked spectrophotometrically and by electrophoresis through a 1.5\% agarose gel following formamide denaturation. $2 \mu \mathrm{g}$ of polyA tailed mRNA was reverse transcribed using ReverseAid First Strand cDNA Synthesis Kit (Thermo Scientific, USA) with an oligo (dT) ${ }_{18}$ primer. For cDNA synthesis of miRNAs, $500 \mathrm{ng}$ of total RNA, including miRNAs and small RNAs, was polyadenylated and subsequently reverse transcribed with universal RT primer using NCode miRNA First-Strand cDNA Synthesis Kit (Invitrogen, USA) according to manufacturer's instruction. Specific forward primers were designed for each miRNA based on the whole sequence of miRNAs, and reverse primers were provided by NCode miRNA cDNA Kit based on universal RT primer.

\section{Quantitative real time PCR (qPCR)}

Six mRNA (actin, HSP-70, HSP-90, ATK, IGFR, and GYS) and four miRNAs (mir-7, mir-34, mir-317, mir-375) were used in qPCR analyses. Glyceraldehyde-3-phosphate dehydrogenase (GAPDH) and the small nucleolar RNA (snoRNA), U51, were used as normalized control transcripts for mRNA and miRNA, respectively. qPCR analyses were carried out on the BioRad CFX96 C1000 Touch Systems. Each reaction contained 10 or $5 \mathrm{ng}$ template cDNA, $500 \mathrm{nM}$ or $300 \mathrm{nM}$ of each primer, and $5 \mu$ l of iTaq universal SYBR Green Supermix (BioRad) in a final volume of $10 \mu \mathrm{l}$ for mRNA and miRNA respectively. Reactions was conducted in triplicate, including negative controls, and all biological replicates were repeated twice. Cycling parameters for both mRNAs and miRNAs were $95^{\circ} \mathrm{C}$ for $30 \mathrm{sec}$ followed by 40 cycles of $90^{\circ} \mathrm{C}$ for $5 \mathrm{sec}$ and $30 \mathrm{sec}$ at $55^{\circ} \mathrm{C}$ for mRNAs, $58.5^{\circ} \mathrm{C}$ for mir34, and $53^{\circ} \mathrm{C}$ for all remaining miRNAs. Melt curves 
carried out at the end of the amplification cycle confirmed the absence of nonspecific amplification and primer dimers. A seven-point standard curve of a ten-fold dilution series was included in each run and used to calculate amplification efficiency for each primer set. Primer sequences for mRNAs, and the reference transcripts were selected using Primer3Plus software (Andreas et al. 2007) and are given in Table 1. Primer specificity was confirmed using BLASTn against the D. magna genome.

To identify the sequence of D. magna U51, U51 sequences from related model species were selected for nucleotide BLAST search against a small RNA database of Daphnia magna (unpublished data). The BLAST hit with $100 \%$ identity was used to generate a primer set for qPCR. Fold-change differences between exposure times of kairomone were calculated based on expression of $t_{0}$ of initial time point using $\Delta \Delta \mathrm{Ct}$ (cycle threshold) method and normalized to expression of reference transcripts. Only data with amplification efficiencies between $89 \%$ and $99 \%$ were used for analysis. The BioRad CFX96 C1000 Touch Systems software automatically set the baseline and threshold settings used for Ct analysis. Statistical significance of differential expression of target genes was determined using One way ANOVA Tukey HST test.

Table 1: The primer names, forward and reverse primer sequences, accession numbers for mRNAs, miRNAs and reference genes. 


\begin{tabular}{|c|c|c|c|}
\hline Primer Name & $\begin{array}{l}\text { Forward and reverse primers }\left(5^{\prime}\right. \\
\left.\text { to } 3^{\prime}\right)\end{array}$ & Accession Number & Reference \\
\hline Actin & $\begin{array}{l}\text { F: } \\
\text { CCACACTGTCCCCATTTATGAA } \\
\text { R: CGCGACCAGCCAAATCC }\end{array}$ & GenBank: AJ292554.1 & $\begin{array}{l}\text { Heckmann } \\
\text { et al. } 2006\end{array}$ \\
\hline HSP-70 & $\begin{array}{l}\text { F: AACTGTGGGAGGAGTCATGA } \\
\text { R: GAAGCCGTGGAGAAGATCTG }\end{array}$ & $\begin{array}{l}\text { Dapma6txEVm003916t1 } \\
\text { Gene Bank: } \\
\text { GDIP01003724.1 }\end{array}$ & This work \\
\hline HSP-90 & $\begin{array}{l}\text { F: CTCACCGAAGCTGTTGATGA } \\
\text { R: TTCAACTCCCTCCTTGGCTA }\end{array}$ & $\begin{array}{l}\text { Dapma6txEVm002351t28 } \\
\text { GenBank: } \\
\text { GDIP01183915.1 }\end{array}$ & This work \\
\hline $\begin{array}{l}\text { AKT-interacting } \\
\text { protein }\end{array}$ & $\begin{array}{l}\text { F: TTCCCCAGACTGACATTCCT } \\
\text { R: CGATCGGGTCTCCATGTAAC }\end{array}$ & Dapma7bEVm028184t1 & This work \\
\hline $\begin{array}{l}\text { Glycogen synthase } \\
\text { (GYS) }\end{array}$ & $\begin{array}{l}\text { F: AGAATGTGGCCGCGATAATT } \\
\text { R: GCCAAACATGTGAGTGCATC }\end{array}$ & Dapma7bEVm023673t1 & This work \\
\hline $\begin{array}{l}\text { Insulin growth } \\
\text { factor receptor } \\
\text { (IGFR) }\end{array}$ & $\begin{array}{l}\text { F: TGGGCATCTTGAAACAACGA } \\
\text { R: CACGAGGTAGACTGCAACTC }\end{array}$ & Dapma7bEVm009863t1 & This work \\
\hline GAPDH & $\begin{array}{l}\text { F: GGCAAGCTAGTTGTCAATGG } \\
\text { R: TATTCAGCTCCAGCAGTTCC }\end{array}$ & GenBank: AJ292555.1 & $\begin{array}{l}\text { Heckmann } \\
\text { et al. } 2006\end{array}$ \\
\hline dma-miR-7 & $\begin{array}{l}\text { F: } \\
\text { TGGAAGACTAGTGATTTTGTTGT }\end{array}$ & Not available & $\begin{array}{l}\text { Ünlü et al. } \\
2015\end{array}$ \\
\hline dma-miR-317 & $\begin{array}{l}\text { F: } \\
\text { GAACACAGCTGGTGGTATCTCA }\end{array}$ & Not available & $\begin{array}{l}\text { Ünlü et al. } \\
2015\end{array}$ \\
\hline dma-miR-34 & $\begin{array}{l}\text { F: } \\
\text { TGGCAGTGTGGTTAGCTGGTTG }\end{array}$ & Not available & $\begin{array}{l}\text { Ünlü et al. } \\
2015\end{array}$ \\
\hline dma-miR-375 & $\begin{array}{l}\text { F: } \\
\text { TTTGTTCGTTCGGCTCGCGTTA }\end{array}$ & Not available & $\begin{array}{l}\text { Ünlü et al. } \\
2015\end{array}$ \\
\hline snRNA - U51 & F: ATCAAATCACCATCTTTCGGC & Not available & $\begin{array}{l}\text { Ünlü et al. } \\
2015\end{array}$ \\
\hline
\end{tabular}

\section{miRNA target prediction and functional annotation}

As chromosomal mapping and miRNA target prediction platforms are not yet available for D. magna, prediction of miRNA and their potential target mRNAs were determined using the homology of D. magna mRNAs with that of sequences obtained from the Drosophila RNA seq database in Fly Base (http://flybase.org). Prediction of putative miRNAs for D. magna genes were selected based on Drosophila genes showing high homology with the $6 \mathrm{mRNAs}$ examined from D. magna using TargetScan 7.2 (http://www.targetscan.org/fly_72/) (Agarwal et al. 2018) and PicTar (https://pictar.mdc-berlin.de) (Grün et al. 2005). From these, four predicted miRNAs were selected for further analysis as they were also present in the Daphnia miRNAs identified by Ünlü et al. 2015. 
Functional roles for putative miRNA targets were determined by analyzing functional annotation data for each target gene. In order to cluster target genes using their GO, COG and KEGG annotation information, the Entrez gene IDs were retrieved using BioMart data mining tool (Durinck et al. 2009). Online Uniprot Retrieve/ID mapping tool was used for interconversion of Entrez gene ID, COG and KEGG accessions (UniProt Consortium, 2018). GO annotation data was obtained by screening GO database for given uniport IDs for each target genes. Data processing and analysis were carried out using custom Perl codes (Ünlü et al., 2015).

\section{Abbreviations}

ATK: AKT-interacting protein; COG: Clusters of Orthologous Groups of proteins; DVM: diurnal vertical migration; GAPDH: glyceraldehyde 3-phosphate dehydrogenase; GYS: Glycogen synthase; GO: Gene Ontology; HSP: heat shock protein; IGFR: Insulin growth factor receptor; KEGG: Kyoto Encyclopedia of Genes and Genomes; miRNA: microRNA; qPCR: quantitative real-time polymerase chain reaction

\section{Declarations}

\section{Acknowledgements}

We would like to thank the Bolu Abant İzzet Baysal University and Mississippi State University Department of Biological Sciences for their support.

\section{Funding}

This study was supported by Bolu Abant İzzet Baysal University Scientific Research Projects (BAIBU-BAP, Project No: 2017.03.01.1151). The funding body does not have a role in the design of the study, data collection, analysis, interpretation of data, writing the manuscript, nor the decision to publish.

\section{Availability of data and materials}

The datasets used and/or analysed during the current study are available from the corresponding author on reasonable request

\section{Authors' contributions}

MT conceived and designed the research design, with technical assistance provided by DMG. MT collected and prepared all biological samples, performed the qRT-PCR experiments, performed the statistical analysis and putative mRNA targets identification, and wrote the first draft of the manuscript. 
ESU carried out all computational analyses including GO, COG, and KEGG characterization. All authors contributed to the analysis of the data, writing of the manuscript and read and approved the final manuscript.

\section{Ethics approval and consent to participate}

Ethic committee of Bolu Abant Baysal University ethically justified the project. Invertebrate and vertebrate animals used in the projects are not protected species. The research does not involve destructive sampling techniques damaging to the environment.

\section{Consent for publication}

Not applicable

\section{Competing interests}

The authors declare that they have no competing interests.

\section{References}

Andreas U, Nijveen H, Rao X, Bisseling T, Geurts R, Jack AM. Leunissen: Primer3Plus, an enhanced web interface to Primer3 Nucleic Acids Research. 2007; 35: W71-W74; doi:10.1093/nar/gkm306

Agarwal W, Subtelny AO, Thiru P \& Bartel DP. Predicting microRNA targeting efficacy in Drosophila. Genome Biology. 2018; 19 (152). doi: 10.1186/s13059-018-1504-3.

Badisco L, Wielendaele PV, Broeck JV. Eat to reproduce: a key role for the insulin signaling pathway in adult insects. 2013; 4: 202. Doi: 10.3389/fphys.2013.00202

Carthew RW, Sontheimer EJ. Origins and mechanisms of miRNAs and siRNAs. Cell. 2009; 136: 642- 55. doi: 10.1016/j.cell.2009.01.035 PMID: 19239886.

Chen S, Nichols KM, Poynton HC, Sepúlveda MS. MicroRNAs are involved in cadmium tolerance in Daphnia pulex. Aquatic Toxicology. 2016; 175: 241-248.

Colbourne JK, Pfrender ME, Gilbert D, Thomas WK, Tucker A, Oakley TH. The ecoresponsive genome of Daphnia pulex. Science. 2011; 331(6017): 555-561. doi: 10.1126/science.1197761 PMID: 21292972 
Coucheron, DG, Wojewodzic, MW, Bøhn, T. MicroRNAs in Daphnia magna identified and characterized by deep sequencing, genome mapping and manual curation. Scientific Reports. 2019; 9: 15945. doi:10.1038/s41598-019-52387-z

Durinck S, Spellman P, Birney E, Huber W. Mapping identifiers for the integration of genomic datasets with the R/Bioconductor package biomaRt. Nature Protocols. 2009; 4: 1184-1191.

Dodson SI, Havel JE. Indirect prey effects: Some morphological and life history responses of Daphnia pulex exposed to Notonecta undulata. Limnol. Oceanogr. 1998; 33:1274-1285.

de Meester, L, Cousyn, $\mathrm{C}$. The change in phototactic behaviour of a Daphnia magna clone in the presence of fish kairomones: the effect of exposure time. Hydrobiologia. 1997; 360: 169-175.

Effertz C, von Elert E. Light intensity controls anti-predator defences in Daphnia: the suppression of lifehistory changes. Proc Biol Sci. 2014; 281: 20133250. http://dx.doi.org/10.1098/rspb.2013.3250

Haap T, Köhler HR. Cadmium tolerance in seven Daphnia magna clones is associated with reduced hsp70 baseline levels and induction. Aquat Toxicol. 2009; 94(2):131-137. doi:10.1016/j.aquatox. 2009.06.006.

Hardie DG. Organismal carbohydrate and lipid homeostasis. Cold Spring Harb Perspect. Biol. 2012; 4: a006031.

Harris KDM, Bartlett NJ, Lloyd VK. Daphnia as an emerging epigenetic model organism. Genetics Research International. 2012; doi:10.1155/2012/147892.

Hearn J, Chow FWN, Barton H, Tung M, Wilson PJ, Blaxter M, Little TJ. Daphnia magna microRNAs respond to nutritional stress and ageing but are not transgenerational. Molecular Ecology. 2018; 27 (6): 1402-1416.

Heckmann L-H, Connon R, Hutchinson TH, Maund SJ, Sibly RM, Callaghan A. Expression of target and reference genes in Daphnia magna exposed to ibuprofen. BMC Genomics. 2006; 7:175. doi:10.1186/1471-2164-7-175

Hu J, Lin C, Liu M, Tong Q, Xu S, Wang D, Zhao Y. Analysis of the microRNA transcriptome of Daphnia pulex during aging. Gene. 2018; 664: 101-110.

Hotamisligil GS and Davis RJ. Cell Signaling and Stress Responses. Cold Spring Harbor Laboratory Press. 2016; doi: 10.1101/cshperspect.a006072.

Grün D, Wang, Y, Langenberger D, Gunsalus KC, Rajewsky N. microRNA target predictions across seven Drosophila species and comparison to mammalian targets. Plos Computational Biology, 2005; https://doi.org/10.1371/journal.pcbi.0010013 
Isik M, Blackwell TK, Berezikov E. MicroRNA mir-34 provides robustness to environmental stress response via the DAF-16 network in C. elegans. Scientific Reports. 2016; 6:36766.

Jain S, Rana V, Tridibes A, Sunil S, Bhatnagar R. Dynamic expression of miRNAs across immature and adult stages of the malaria mosquito Anopheles stephensi. Parasites and Vectors. 2015; 8:179

Jansen M, Vergauwen L, Vandenbrouck T, Knapen D, Dom N, Spanier KI, Cielen A, De Meester L. Gene expression profiling of three different stressors in the water flea Daphnia magna. Ecotoxicology. 2013; 22: 900-914.

Judice CC, Bourgard C, Kayano ACAV, Albrecht L, Costa FTM. MicroRNAs in the Host-Apicomplexan Parasites Interactions: A Review of Immunopathological Aspects. Frontiers in Cellular and Infection Microbiology. 2016; https://doi.org/10.3389/fcimb.2016.00005.

Klüttgen B, Dülmer U, Engels M, Ratte HT. ADaM, an artificial freshwater for the culture of zooplankton. Water Research. 1994; 28:743-746.

Kregel KC. Heat shock proteins: modifying factors in physiological stress responses and acquired thermotolerance. J Appl Physiol (Bethesda, Md.: 1985). 2002; 92(5): 2177-2186.

doi:10.1152/japplphysiol. 01267.2001.

Krebs RA, Feder ME. Deleterious consequences of Hsp70 overexpression in Drosophila melanogaster larvae. Cell Stress Chap. 1997; 2: 60-71.

$\mathrm{Li}, \mathrm{X}$, Cassidy JJ, Reinke CA, Fischboeck S, Carthew, RW. A microRNA imparts robustness against environmental fluctuation during development. Cell. 2009; 137:273-282.

Marzi MJ, Ghini F, Cerruti B, Pretis S, Bonetti P, Giacomelli C, Gorski MM, Kress T, Pelizzola M, Muller H, Amati B, Nicassio F. Degradation dynamics of microRNAs revealed by a novel pulse-chase approach. Genome Research. 2016; 26:554-565.

Meester L, De Dawidowicz P, Loose C, van Gool E. Ecology and evolution of predator-induced behavior of zooplankton: depth selection behavior and diel vertical migration. The Ecology and Evolution of Inducible Defenses (Tollrian R, Harvell D, Eds) Princeton Univ Press; 1999.

Miyakawa H, Imai M, Sugimoto N, Ishikawa Y, Ishikawa A, Ishigaki H, Okada Y, Miyazaki S, Koshikawa S, Cornette R, Miura T. Gene up-regulation in response to predator kairomones in the water flea, Daphnia pulex. BMC Developmental Biology. 2010; 10: 45.

Nollen EAA and Morimoto RI. Chaperoning signaling pathways: molecular chaperones as stress-sensing 'heat shock' proteins. Journal of Cell Science. 2002; 115: 2809-2816.

Otte KA, Thomas Fröhlich T, Arnold GJ, Laforsch C. Proteomic analysis of Daphnia magna hints at molecular pathways involved in defensive plastic responses. BMC Genomics. 2014; 15:306 
Pauwels K, Stoks R, de Meester L. Coping with predator stress: interclonal differences in induction of heat-shock proteins in the water flea Daphnia magna. J Evol Biol. 2005; 18(4): 867-872. doi:10.1111/j.1420-9101.2005.00890.x.

Pijanowska J, Kloc M. Daphnia response to predation threat involves heat- shock proteins and the actin and tubulin cytoskeleton. Genesis. 2004; 38: 81-86.

Price C, Chen J. MicroRNAs in cancer biology and therapy: Current status and perspecives. Genes and Diseases. 2014; 1: 53-63. PMID: 25473652

Reynolds CS. Daphnia: Development of model organism in ecology and evolution. Freshwater Reviews. 2011; 4: 85-87.

Rozenberg A, Parida M, Leese F, Weiss LC, Tollrian R, Manak JR: Transcriptional profiling of predatorinduced phenotypic plasticity in Daphnia pulex. Front Zool. 2015; 12:18.

Schwarzenberger A, Courts C, von Elert E. Target gene approaches: Gene expression in Daphnia magna exposed to predator-borne kairomones or to microcystin-producing and microcystin-free Microcystis aeruginosa. BMC Genomics. 2009; 10:527.

Silbermann R, Tatar M. Reproductive costs of heat shock protein in transgenic Drosophila melanogaster. Evolution. 2000; 54: 2038-2045.

Sorensen JG, Kristensen TN, Loeschcke V. The evolutionary and ecological role of heat shock proteins. Ecol Lett. 2003; 6:1025-1037. doi:10.1046/j. 1461-0248.2003.00528.x

Stark A, Brennecke J, Russell RB, Cohen SM. Identification of Drosophila microRNA targets. PLoS Biology 2003; 1(3): e60. doi:10.1371/journal.pbio.

Stibor H. Predator induced life-history shifts in a freshwater cladoceran. Oecologia. 1992; 92: 162-165.

Tollrian R. Predator-induced morphological defenses: costs, life history shifts, and maternal effects in Daphnia pulex. Ecology. 1995; 76: 1691-1705.

Ünlü ES, Gordon DM, Telli M. Small RNA sequencing based identification of miRNAs in Daphnia magna. PLoS One. 2015; 10 (9); e0137617.

UniProt Consortium. UniProt: a worldwide hub of protein knowledge. Nucleic Acids Research. 2018; 47 (D1): D506-D515.

\section{Figures}



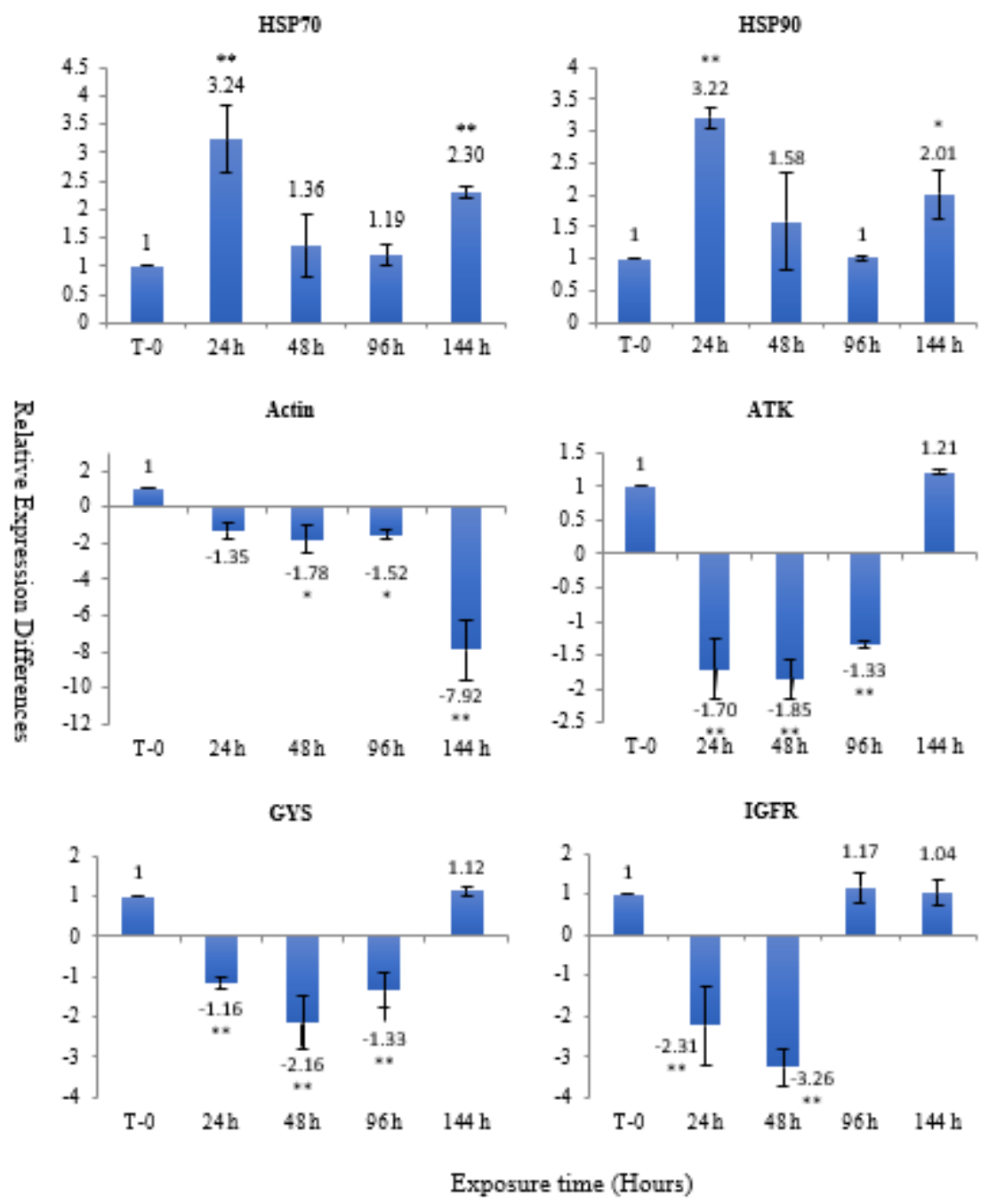

\section{Figure 1}

Relative gene expression differences with kairomone exposure as determine by qRT-PCR for select mRNA transcripts in D. magna. Data represents the mean \pm SEM $(n=6)$. Statistical significance was determined using One way ANOVA Tukey HST test; * $p<0.05$, ** $p<0.01$. 


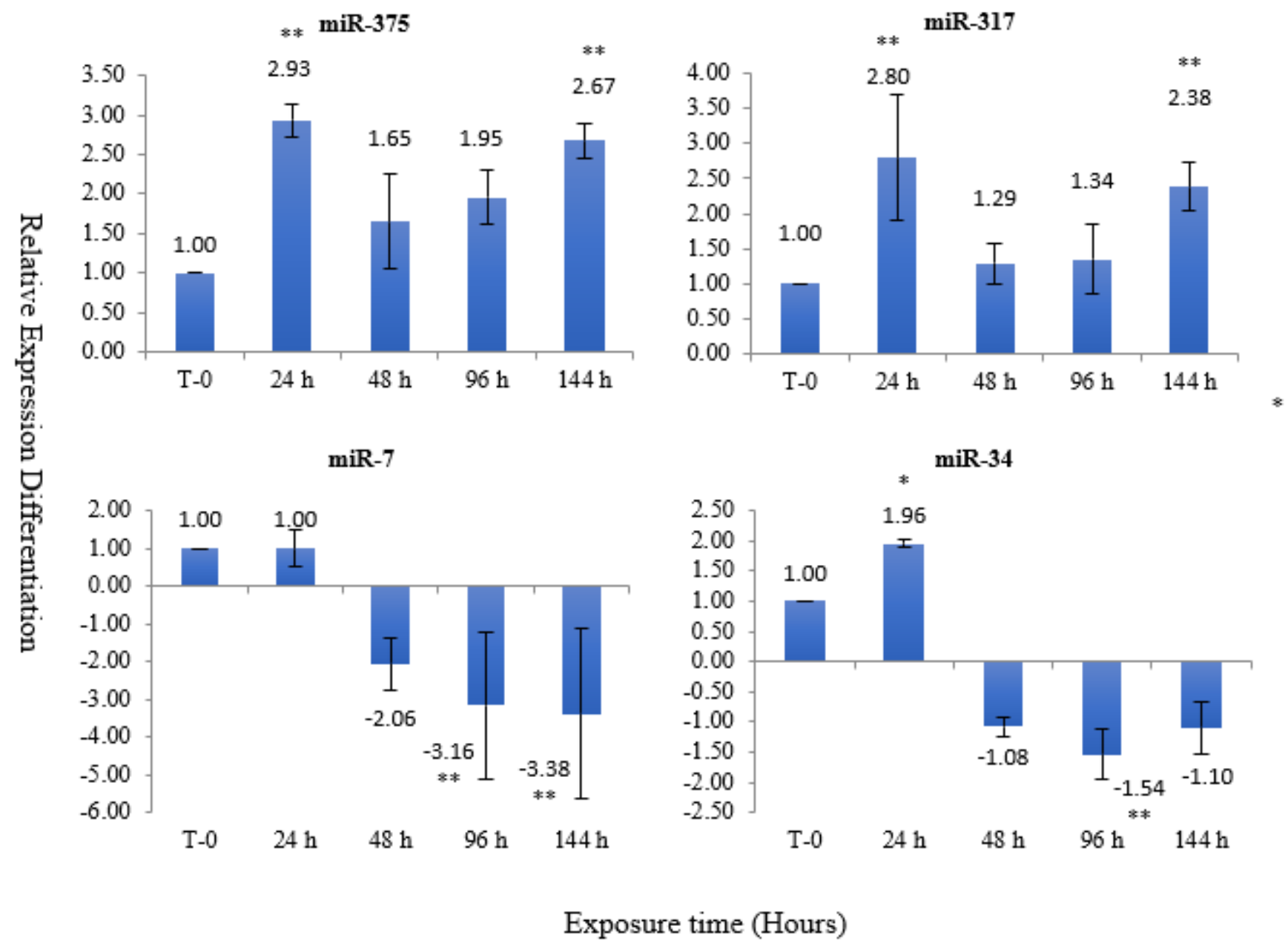

Figure 2

Relative gene expression differences with kairomone exposure as determine by qRT-PCR for select miRNA transcripts in D. magna. Data represents the mean \pm SEM $(n=6)$. Statistical significance was determined using One way ANOVA Tukey HST test; ${ }^{*} p<0.05,{ }^{* \star} p<0.01$.

\section{Supplementary Files}

This is a list of supplementary files associated with this preprint. Click to download.

- AddtionalFigure1COG.tif

- NC3RsARRIVEGuidelinesChecklistfillable.pdf

- AddtionalFigure2GO.tif

- AddtionalFigure3KEGG.tif

- AdditionalTable1.xIsx 